EPJ Web of Conferences 20, 05003 (2012)

DOI: $10.1051 /$ epjconf/20122005003

(c) Owned by the authors, published by EDP Sciences, 2012

\title{
Status of J-PARC K1.8 Beam Line
}

Kiyoshi Tanida ${ }^{a}$ for J-PARC K1.8 beamline group

Department of Physics, Seoul National University

\begin{abstract}
The commissioning of the $\mathrm{K} 1.8$ beam line in the hadron hall of J-PARC has been going very well. In November 2011, the first physics data was taken for E19 experiment, which is the day-one experiment in the hadron hall of J-PARC. A preliminary result of E19 exhibits no peak for pentaquark $\Theta^{+}$around $1540 \mathrm{MeV}$.
\end{abstract}

\section{Overview of J-PARC}

J-PARC (Japan Proton Accelerator Research Complex) is an accelerator complex constructed in Tokai, Japan (Fig. 1), and provides world strongest proton beam of an order of $1 \mathrm{MW}$ up to $50 \mathrm{GeV}$. Utilizing its strong beam, variety of researches, such as protein crystallography, transmutation of nuclear wastes, as well as nuclear and particle physics are planned.

\subsection{Hadron Hall and K1.8 Beam Line}

Most of the nuclear and particle physics experiments are performed in the Hadron Hall (Hadron Experimental Area as shown in top-right corner of Fig. 1). In the Hadron Hall, among the 6 beam lines (see Fig. 2) planned to be constructed, 4 of them (K1.8, K1.8BR, K1.1BR, and KL) are now available.

a e-mail: tanida@phya.snu.ac.kr

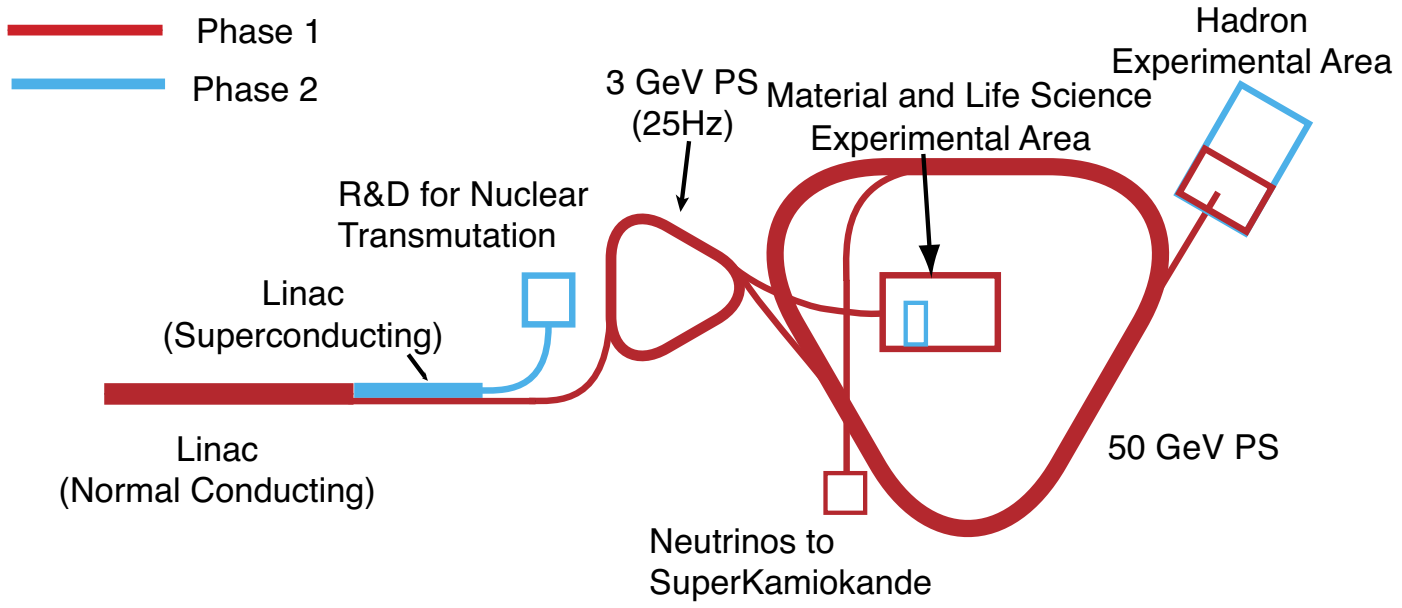

Fig. 1. Schematic layout of the J-PARC facility. Construction of the Phase 1 facility is completed, and Phase 2 facility is to be approved by the government.

This is an Open Access article distributed under the terms of the Creative Commons Attribution-Noncommercial License 3.0, which permits unrestricted use, distribution, and reproduction in any noncommercial medium, provided the original work is properly cited. 


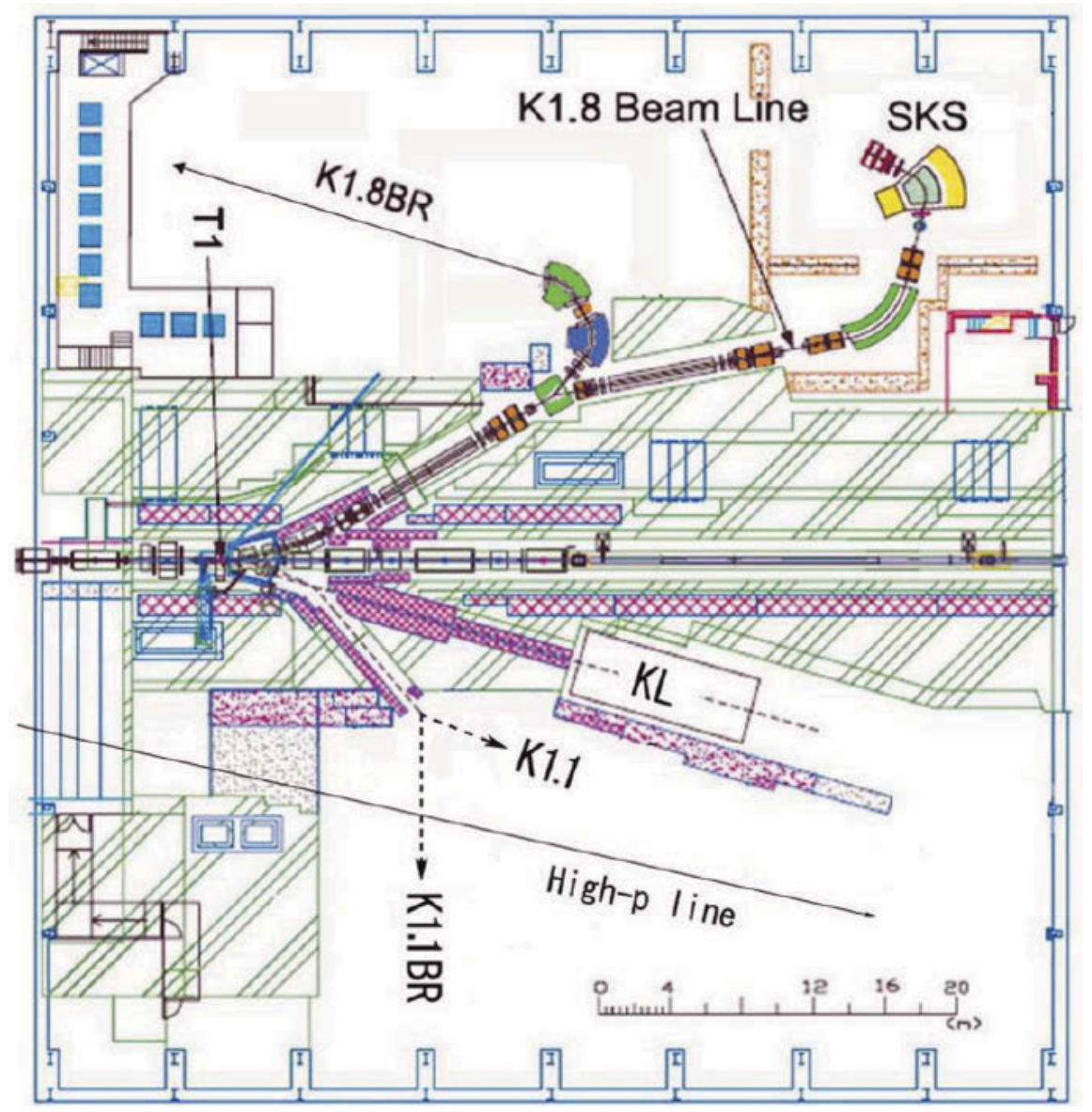

Fig. 2. Plan view of the Hadron Hall. Six secondary beam lines are planned to be constructed in the Hall.

The K1.8 beam line can provide separated charged particles up to $2 \mathrm{GeV} / \mathrm{c}$, and mostly used for spectroscopic experiments with kaon beams. The designed performance of the K1.8 beam line is summarized in Table 1. A notable feature is that a high-purity kaon beams can be obtained with high intensity thanks to the double electrostatic separators. Also, the last QQDQQ magnets form a highresolution spectrometer, which gives an expected momentum resolution of about $0.6 \mathrm{MeV} / \mathrm{c}$ for 1.8

Table 1. Design performance of the K1.8 beam line.

\begin{tabular}{|c|c|c|c|}
\hline & Phase I & Phase II \\
\hline \multirow{2}{*}{\multicolumn{2}{|c|}{$\begin{array}{r}\text { Primary proton beam energy } \\
\text { intensity }\end{array}$}} & $30 \mathrm{GeV}$ & $50 \mathrm{GeV}$ \\
\hline & & $2.0 \times 10^{14} / \operatorname{spill}(9 \mu \mathrm{A})$ & $3.3 \times 10^{14} /$ spill $(15 \mu \mathrm{A})$ \\
\hline \multirow{2}{*}{\multicolumn{2}{|c|}{$\begin{array}{l}\text { Length } \\
\text { Acceptance }\end{array}$}} & \multicolumn{2}{|c|}{$45.97 \mathrm{~m}$} \\
\hline & & \multicolumn{2}{|c|}{$1.4 \mathrm{msr} \%$} \\
\hline \multirow[t]{3}{*}{$K^{-}$intensity at } & $1.8 \mathrm{GeV} / \mathrm{c}$ & $1.4 \times 10^{6} /$ spill & $6.6 \times 10^{6} /$ spill \\
\hline & $1.5 \mathrm{GeV} / \mathrm{c}$ & $5.4 \times 10^{5} /$ spill & $2.7 \times 10^{6} /$ spill \\
\hline & $1.1 \mathrm{GeV} / \mathrm{c}$ & $8.0 \times 10^{4} /$ spill & $3.8 \times 10^{5} /$ spill \\
\hline \multirow{2}{*}{\multicolumn{2}{|c|}{$\begin{array}{l}K^{-} /\left(\pi^{-}+\mu^{-}\right) \text {ratio at } 1.8 \mathrm{GeV} / \mathrm{c} \\
\text { RMS beam size at taroet }\end{array}$}} & \multicolumn{2}{|c|}{$\begin{array}{ll}3.5 & 4.0\end{array}$} \\
\hline & RMS beam size at target & \multicolumn{2}{|c|}{$19.8 \mathrm{~mm}(\mathrm{X}) / 3.2 \mathrm{~mm}(\mathrm{Y})$} \\
\hline
\end{tabular}


Table 2. List of approved experiments to be executed at K1.8 beam line. Details on these experiments are found in proposals available from J-PARC homepage [3].

\begin{tabular}{|c|c|c|}
\hline exp. \# & Title & Spokesperson \\
\hline E03 & Measurement of $X$ rays from $\Xi^{-}$atoms & K. Tanida (Seoul Nat. U.) \\
\hline E05 & $\begin{array}{l}\text { Spectroscopic study of } \Xi \text {-hypernucleus, }{ }_{\Xi}^{12} \mathrm{Be} \text {, } \\
\text { via the }{ }^{12} \mathrm{C}\left(K^{-}, K^{+}\right) \text {reaction }\end{array}$ & T. Nagae (Kyoto U.) \\
\hline E07 & $\begin{array}{l}\text { Systematic study of double strangeness system with } \\
\text { an emulsion-counter hybrid method }\end{array}$ & $\begin{array}{l}\text { K. Imai (JAEA) } \\
\text { K. Nakazawa (Gifu U.) } \\
\text { H. Tamura (Tohoku U.) }\end{array}$ \\
\hline E10 & $\begin{array}{l}\text { Production of neutron-rich } \Lambda \text {-hypernuclei with the } \\
\text { double charge-exchange Reaction }\end{array}$ & A. Sakaguchi (Osaka U.) \\
\hline E13 & Gamma-ray spectroscopy of light hypernuclei & H. Tamura (Tohoku U.) \\
\hline E18 & $\begin{array}{l}\text { Coincidence measurement of the weak decay of }{ }_{\Lambda}^{12} \mathrm{C} \\
\text { and the three-body weak interaction process }\end{array}$ & $\begin{array}{l}\text { H.C. Bhang (Seoul Nat. U.) } \\
\text { H. Outa (RIKEN) } \\
\text { H. Park (KRISS) }\end{array}$ \\
\hline E19 & $\begin{array}{l}\text { High-resolution search for } \Theta^{+} \text {pentaquark in } \pi^{-} p \rightarrow K^{-} X \\
\text { reactions }\end{array}$ & M. Naruki (KEK) \\
\hline E27 & $\begin{array}{l}\text { Search for a nuclear } \bar{K} \text { bound state } K^{-} p p \text { in the } d\left(\pi^{+}, K^{+}\right) \\
\text {reaction }\end{array}$ & T. Nagae (Kyoto U.) \\
\hline
\end{tabular}

$\mathrm{GeV} / \mathrm{c}$ kaons. Utilizing those features, a variety of experiments are planned, as shown in Table 2. Most of these experiments study nuclear systems with strangeness.

The beam line is equipped with trigger hodoscopes (BH1 and BH2) that measure time-of-flight of particles, and cherenkov counters (GC, BAC1 and BAC2) that identify particles at the trigger level. MWPCs and drift chambers are uesd for tracking and momentum measurement of beam particles. These detectors are designed to be operated under the high rate beam up to $10^{7} \mathrm{~Hz}$.

The particles produced at the target are analyzed by SKS (Superconducting Kaon Spectrometer), which were used in KEK-PS in Tsukuba for a long time [1]. It has both large acceptance (solid angle $100 \mathrm{msr})$ and good momentum resolution $(\delta p / p \sim 0.1 \%)$, and is an ideal spectrometer for hypernuclear studies. In J-PARC, in order to meet different requirements of various experiments, the SKS magnet is arranged to be movable by air floatation system [2]. A picture of K1.8 beam line together with SKS is shown in Fig. 3.

\section{Performance of the $\mathrm{K} 1.8$ beam line}

The construction of K1.8 beam line was completed in July 2009. Since then, intermittent beam times were used for commissioning of the beam line, concurrent with the accelerator studies, until October 2010. During this period, performance of the beam line and detectors were studied, and all components were confirmed to work well. For example, kaons can be clearly separated from pions even at 1.8 $\mathrm{GeV} / \mathrm{c}$ as shown in Fig. 4 . The bad $K^{-} / \pi^{-}$ratio of about 0.14 was due to the lower separator voltage $( \pm 200 \mathrm{kV}$ with the nominal separator voltage of $\pm 350 \mathrm{kV})$, and thus is not a problem. The yield of $K^{-}$ at $1.8 \mathrm{GeV} / \mathrm{c}$ was $2.5 \times 10^{4} /$ spill for the primary proton intensity of $3.5 \times 10^{12} / \mathrm{spill}$, and is consistent with the expectation.

For the spectrometer system, we took $p\left(\pi^{-}, K^{+}\right) \Sigma^{-}$and $p\left(\pi^{+}, K^{+}\right) \Sigma^{+}$data for calibration and crosscheck. The results are shown in Fig. 5. A peak for $\Sigma^{ \pm}$was clearly seen and the missing mass resolution of $1.9 \mathrm{MeV} / \mathrm{c}^{2}$ (FWHM) was achieved. Differential cross-section was also measured and found to be consistent with past measurement [4] for $\Sigma^{+}$. Here, the performance of the system was confirmed.

Current issues are mostly related to the performance of the primary beam. The beam intensity was typically $3.5 \times 10^{12} /$ spill during the stable operation with the highest intensity $10^{13} /$ spill was demonstrated. The cycle time was $6 \mathrm{~s}$, so the average intensity was $0.09 \mu \mathrm{A}$, which is still about $1 \%$ of the design value. Although this intensity is enough for experiments using pion beams, for which the 


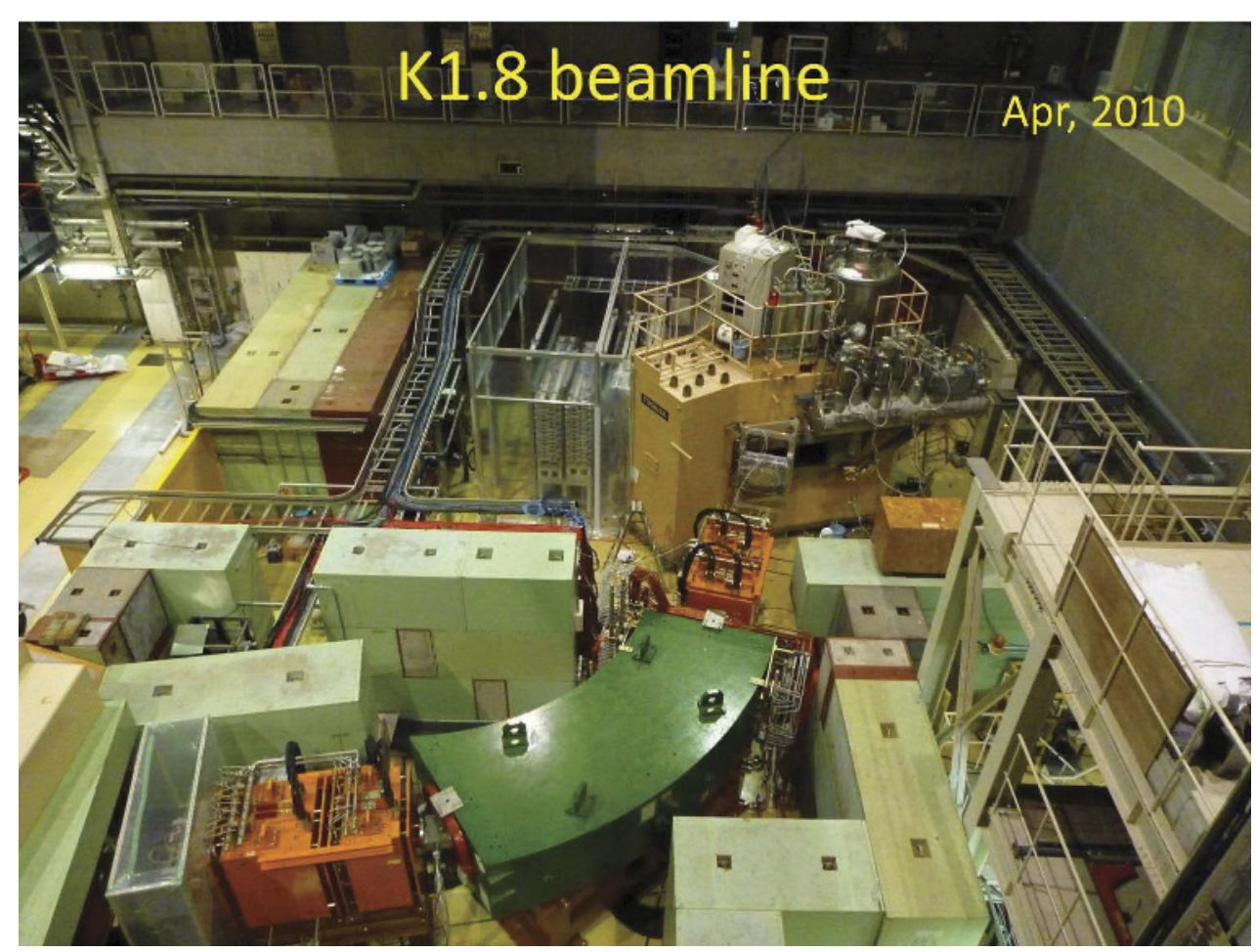

Fig. 3. Picture of K1.8 beam line taken in April 2010.

actual intensity is limited to $\sim 10 \mathrm{MHz}$ by detector performance, we need much higher intensity for experiments usings kaon beams.

For the experiments using pion beams, even more severe issue is the time structure of the beam. Ideally, the extracted beam should have a time structure that the beam is off for a few second and on for another a few second (spill), during which the intensity is constant. However, the actual beam had a spiky structure as shown in Fig. 6. This limits the usable average beam intensity to $1.1 \mathrm{M} \pi / \mathrm{spill}$ ( 1 spill $=2.2 \mathrm{~s}$ ), with the maximum instantaneous intensity being $\sim 10 \mathrm{MHz}$, which is close to our expectation. These issues are gradually improving, and we expect they will be eventually resolved in the coming a few years.

\section{E19 experiment}

In November 2010, the first physics data was taken for E19 experiment, in which a $S=+1$ pentaquark, $\Theta^{+}$was searched for by the $p\left(\pi^{-}, K^{-}\right)$reaction. The first report on the evidence of the $\Theta^{+}$[5] was surprising and had a huge impact in the field of hadron physics. The "discovery" was supported by several collaborations [6-14], but the statistics of all experiments were not sufficient to be conclusive, and later, null results have been reported from several high energy experiments where they searched for $\Theta^{+}$with much higher statistics [15-25]. In addition, some of the initial positive evidences were denied by the same collaboration with higher statistics [26,27]. Thus, the existence of $\Theta^{+}$is still controversial [28]. Therefore, the confirmation of the existence (or non-existence) of the $\Theta^{+}$is urgent and important.

In this situation, a $\Theta^{+}$search was conducted via the $p\left(\pi^{-}, K^{-}\right)$reaction with the experimental resolution of $13.4 \mathrm{MeV}$ FWHM using 1.87 and $1.92 \mathrm{GeV} / \mathrm{c} \pi^{-}$beam at the K2 beam line of the KEK $12 \mathrm{GeV}$ Proton Synchrotron (KEK-PS) [29]. A hint of peak structure, of which width was consistent with the experimental resolution, was observed at the mass of $1530.6_{-1.9}^{+2.2}$ (stat. $)_{-1.3}^{+1.9}$ (syst.) MeV with a possible production cross section of about $1.9 \mu \mathrm{b} / \mathrm{sr}$ (see Fig. 7) for $1.92 \mathrm{GeV} / c$. However the statistical 


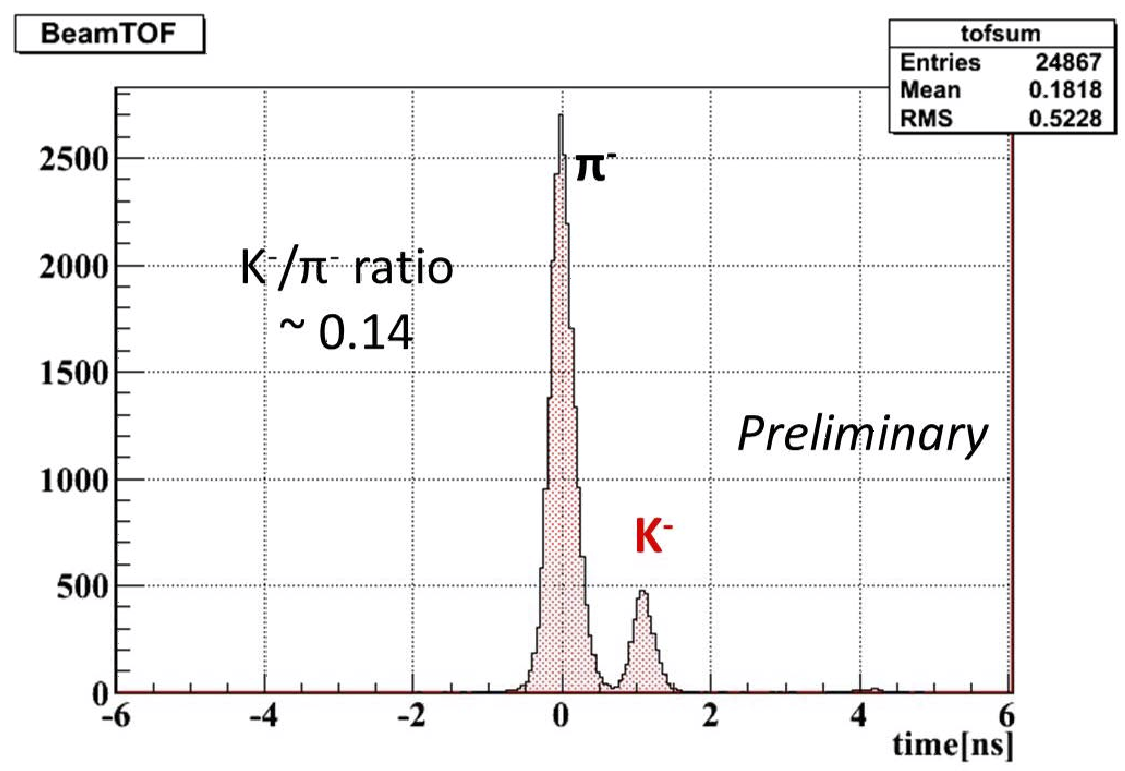

Fig. 4. BH1-BH2 time-of-flight spectrum for negatively charged particles. Flight time for $\pi^{-}$is adjusted to zero. A peak for $K^{-}$is clearly seen.
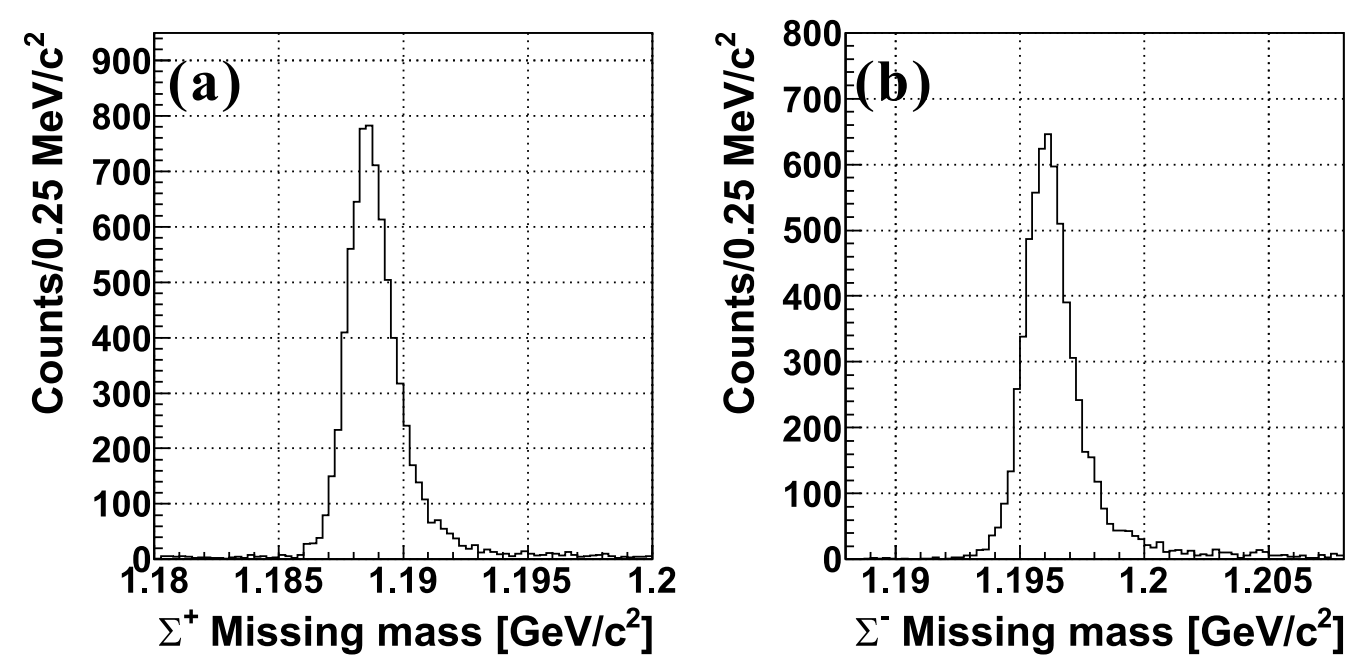

Fig. 5. Missing mass spectrum for $p\left(\pi^{+}, K^{+}\right) \Sigma^{+}$(a) and $p\left(\pi^{-}, K^{+}\right) \Sigma^{-}$(b). The missing mass resolution was obtained to be $1.9 \mathrm{MeV} / \mathrm{c}^{2}$ (FWHM).

significance of the bump is only $2.5 \sim 2.7 \sigma$ which is not sufficient to claim the existence of the $\Theta^{+}$. They have derived the upper limit of the production cross section (averaged over $0^{\circ}$ to $20^{\circ}$ in the laboratory frame) to be $1.6 \mu \mathrm{b} / \mathrm{sr}$ and $2.9 \mu \mathrm{b} / \mathrm{sr}$ at the $90 \%$ confidence level at the beam momentum of 1.87 and $1.92 \mathrm{GeV} / c$, respectively.

In E19, we repeated the same experiment with much higher statistics and much better mass resolution. The plan is to obtain 100 times more statistics than E522, and in the beam time of November 2011 , we accumulated a statistics of $7.8 \times 10^{1} 0$ pions on target (in 156 hours), which is only $5 \%$ of the original plan but still gives $\sim 5$ times larger statistics than KEK-PS E522. Furthermore, an important 
advantage of this experiment is an excellent mass resolution of $2 \mathrm{MeV}$ (FWHM). The high resolution not only gives better signal-to-noise ratio, but also makes it possible to measure the width directly down to $\sim 1 \mathrm{MeV}$ which is near the present limit imposed from the analysis of $K N$ elastic scattering [30]. Such high resolution search was never conducted before, and thus E19 has a good chance to obtain a conclusive result.

A preliminary result is shown in Fig. 8. No peak for Theta ${ }^{+}$was observed. The upper limit is roughly estimated to be around $0.4 \mu \mathrm{b} / \mathrm{sr}$, and thus the previous "hint" reported by E522 is ruled out. Note that the present data has only $\sim 5$ originally planned statistics, and we will further accumulate the data to achieve the sensitivity goal of $75 \mathrm{nb} / \mathrm{sr}$.

\section{Summary}

The construction and commissioning of the K1.8 beam line was successful, and we have just started to take physics data. All components were confirmed to be working well, and present issues are mostly related to the quality and quantity of the primary beam. In the first physics run of E19, $\Theta^{+}$was searched for in the $p\left(\pi^{-}, K^{-}\right)$reaction but the preliminary spectrum shows no peak. The result ruled out the "hint" of a peak observed in KEK-PS E522. We will continue to take data for E19 and for other experiments in the coming runs.

\section{Brief note on damage from the earthquake on March 11, 2011}

On March 11th, 2011, two weeks after the HNP2011 conference, a huge earthquake attacked the northeastern area of Japan, including Tokai village. First of all, the most fortunate thing was that no single persons were injured or dead. Furthermore, No tsunami hit the J-PARC due to the tsunami protection up to 8 meters. Also, major components for the accelerators and experimental facilities, including Hadron Hall received no serious damage thanks to many underpins for the corresponding buildings.

However, all roads around the buildings and utilities, in particular power supplies and cooling water supplies, received significant damage. In addition, new added buildings were dropped by $30 \mathrm{~cm}$ and

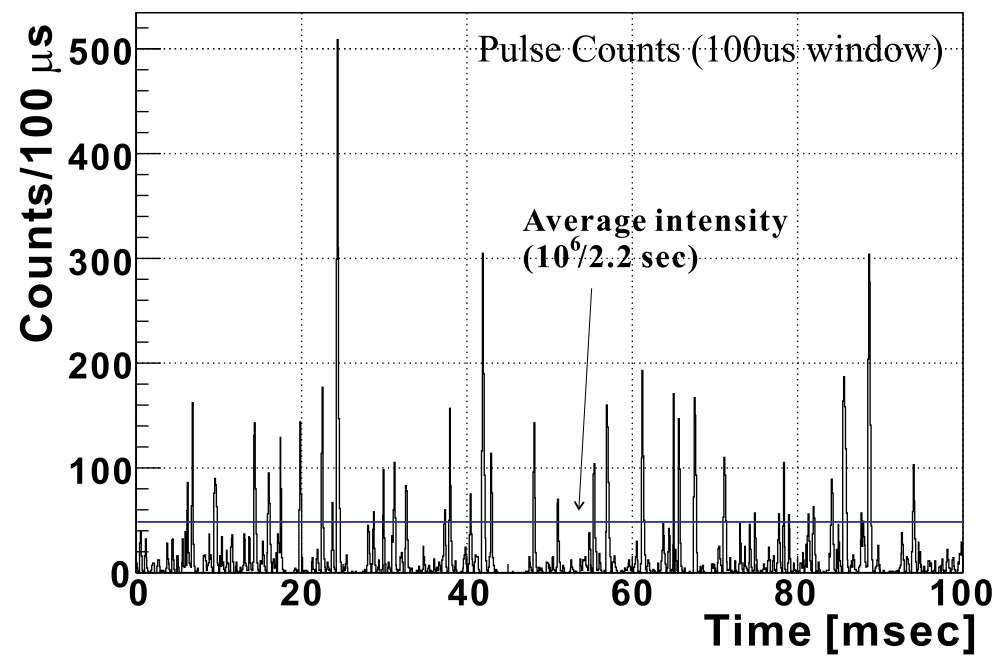

Fig. 6. The beam structure of beam in K1.8 beam line. While the average intensity was 50 particles (mostly pions) per $100 \mu \mathrm{s}$, the instantaneous intensity was sometimes 10 times as high as that. 
they were damaged. For $3 \mathrm{GeV}$ booster ring, both power supplies and cooling water were significantly inclined and damaged. There were many minor damages to various equipments as well.

J-PARC is now trying to recover the road with first priority, as well as many other components. According to the official announcement by J-PARC center, they are trying to resume J-PARC activities by December, 2011. Thus, the anticipated delay of operation is about 1 year.

\section{Acknowledgment}

This work is supported by the BK21 and WCU program of NRF, Korea, and by the grant for scientific research project "Nuclei with Strangeness" from MEXT, Japan.

\section{References}

1. T. Fukuda et al., Nucl. Instr. Meth. A 361 (1995) 485.

2. T. Takahashi, Nucl. Phys. A835 (2010) 88-95.

3. http://j-parc.jp/NuclPart/Proposal_e.html

4. D. J. Candlin et al., Nucl. Phys. B226 (1983) 1.

5. T. Nakano et al., Phys. Rev. Lett. 91 (2003) 012002.

6. DIANA Collaboration, V. V. Barmin et al., Phys. Atom. Nucl. 66 (2003) 1715.

7. CLAS Collaboration, S. Stepanyan et al., Phys. Rev. Lett. 91 (2003) 252001.

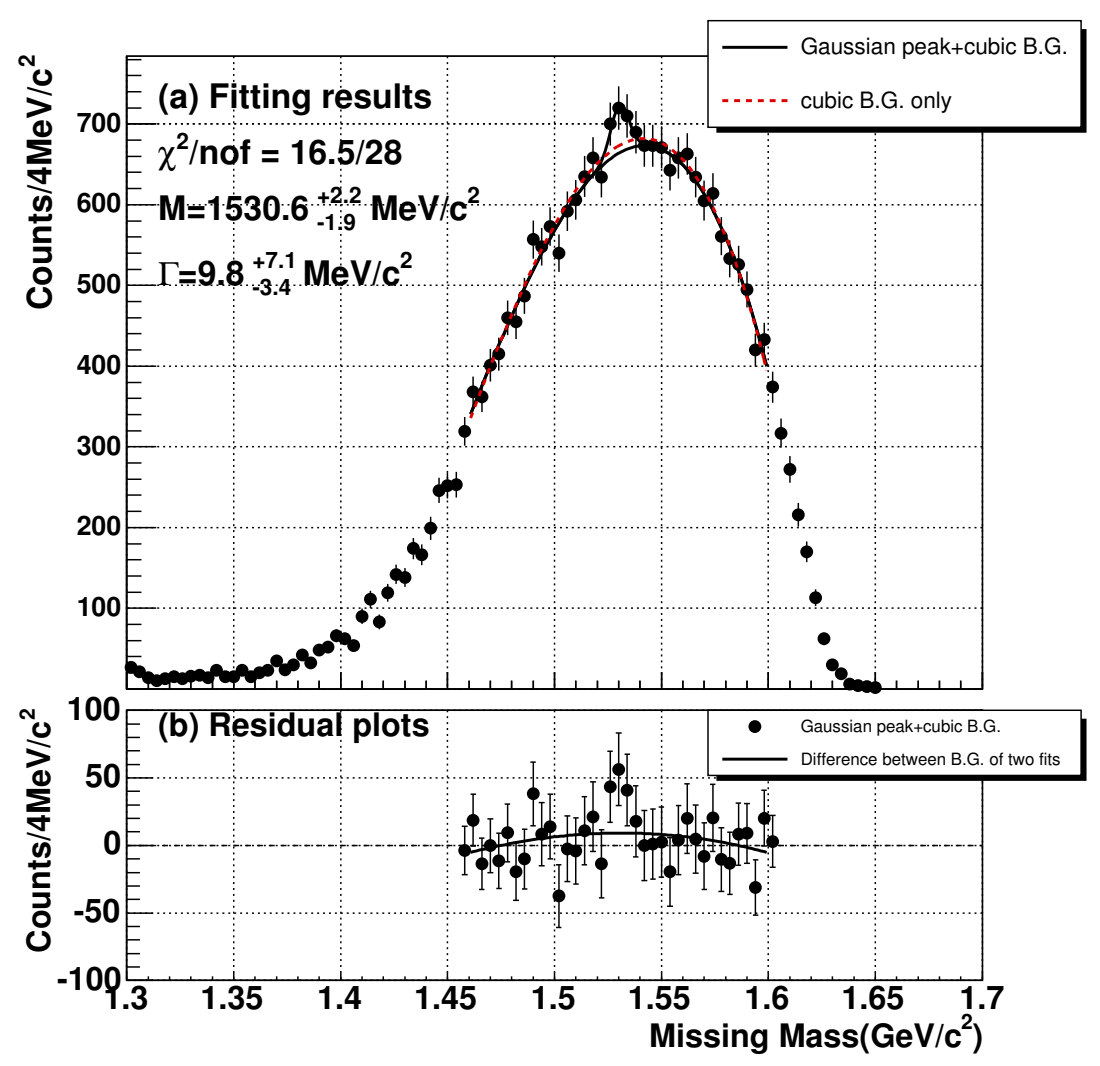

Fig. 7. Missing mass spectrum obtained in E522. A bump structure is seen at $1.53 \mathrm{GeV}$. 


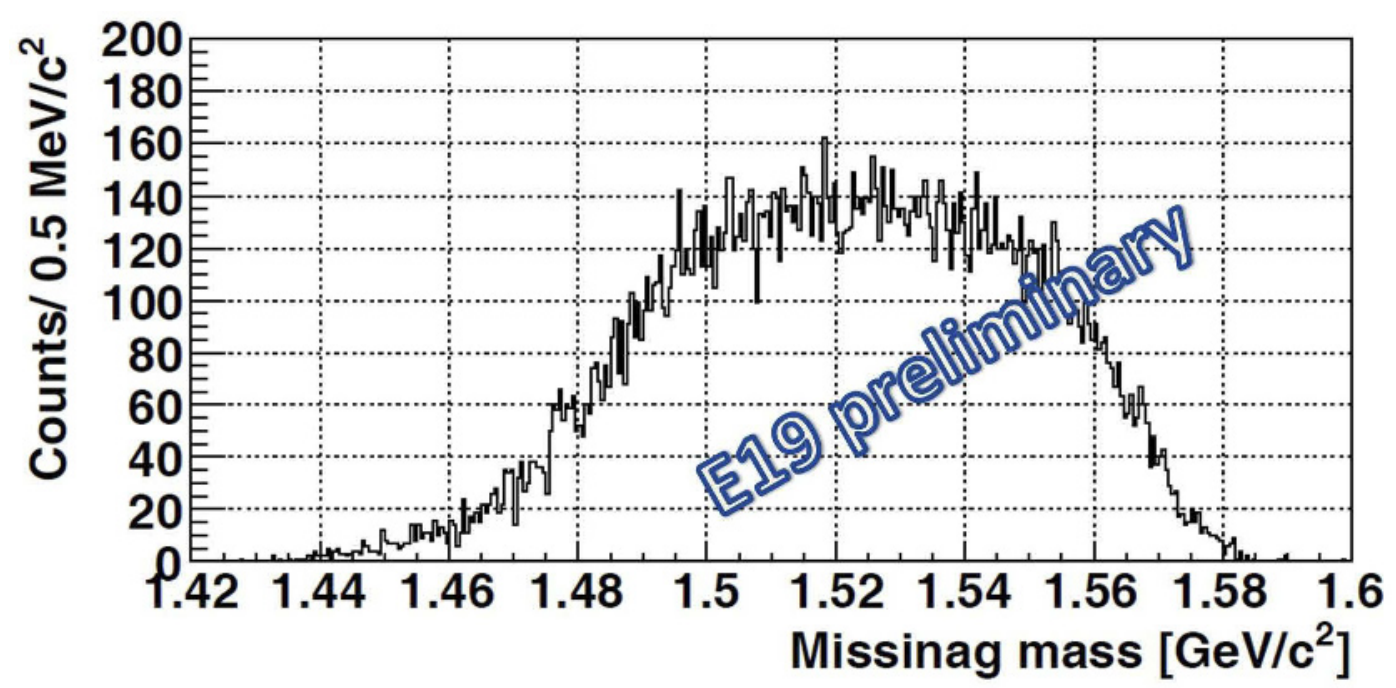

Fig. 8. Missing mass spectrum obtained in E19. No peak was observed for $\Theta^{+}$. Upper limit of the cross section was estimated to be $\sim 0.4 \mu \mathrm{b} / \mathrm{sr}$.

8. CLAS Collaboration, V. Kubarovsky et al., Phys. Rev. Lett. 92 (2004) 032001.

9. SAPHIR Collaboration, J. Barth et al., Phys. Lett B572 (2003) 127.

10. A. E. Asratayn, A. G. Dolgolenko and M. A. Kubantsev, Phys. Atom. Nucl. 67 (2004) 682.

11. HERMES Collaboration, A. Airapetian et al., Phys. Lett. B585 (2004) 213.

12. ZEUS Collaboration, S. Chekanov et al., Phys. Lett. B591 (2004) 7.

13. COSY-TOF Collaboration, M. Abdel-Bary et al., Phys. Lett. B595 (2004) 127.

14. SVD Collaboration, A. Aleev et al., arXiv:hep-ex/0401024.

15. BES Collaboration, J. Z. Bai et al., Phys. Rev. D70 (2004) 012004.

16. BaBar Collaboration, B. Aubert et al., arXiv:hep-ex/0502004.

17. Belle Collaboration, K. Abe et al., arXiv:hep-ex/0409010.

18. ALEPH Collaboration, S. Schael et al., Phys. Lett. B599 (2004) 1.

19. HERA-B Collaboration, I. Abt et al., Phys. Rev. Lett. 92, 212003.

20. SPHINX Collaboration, Yu. M. Antipov et al., Eur. Phys.J. A21 (2004) 455.

21. Hyper-CP Collaboration, M. J. Longo et al., Phys. Rev. D70 (2004) 111101(R).

22. CDF Collaboration, D. O. Litvintsev et al., Nucl. Phys. B (Proc. Suppl.) 142 (2005) 374.

23. FOCUS Collaboration, K. Stenson et al., Int. J. Mod. Phys. A20 (2005) 3745.

24. Belle Collaboration, Phys. Lett. B632 (2006) 173.

25. PHENIX Collaboration, C. Pinkerton et al., J. Phys. G30 (2004) S1201.

26. CLAS Collaboration, M. Battaglieri et al., Phys. Rev. Lett. 96 (2006) 042001.

27. CLAS Collaboration, B. McKinnon et al., arXiv:hep-ex/0603028.

28. K. H. Hicks, Prog. Part. Nucl. Phys. 55 (2005) 647.

29. K. Miwa et al., Phys. Lett. B635 (2006) 72.

30. A. Sibirtsev, J. Haidenbauer, S. Krewalda, and Ulf-G. Meißner, Phys. Lett. B599 (2004) $230 ? 235$. 